(c) 2018 IEEE. Personal use of this material is permitted. Permission from IEEE must be obtained for all other uses, in any current or future media, including reprinting/republishing this material for advertising or promotional purposes, creating new collective works, for resale or redistribution to servers or lists, or reuse of any copyrighted component of this work in other works. 


\title{
Linearly Polarized Shaped Power Pattern Synthesis with Sidelobe and Cross-Polarization Control by using Semidefinite Relaxation
}

\author{
Yanhui Liu, Member, IEEE, Jingjing Bai, Kai Da Xu, Member, IEEE, Zhiyao Xu, Feng Han, \\ Qing Huo Liu, Fellow, IEEE, and Y. Jay Guo, Fellow, IEEE
}

\begin{abstract}
In this Communication, the problem of synthesizing a linearly polarized shaped power pattern with accurate control on both sidelobe and cross-polarization levels is considered. For a user-defined desired polarization direction, the definitions of realizable co-polarization (COP) and cross-polarization (XP) directions for an arbitrary propagation direction in the shaped pattern are presented. With the help of such definitions, the considered problem is formulated as finding appropriate excitations so as to produce a shaped power pattern in which the realizable COP component meets prescribed lower and upper bounds, the realizable XP component and the total power pattern are less than their upper bounds in the regions of interest. The semidefinite relaxation (SDR) method in literature is then extended to solve this vectorial pattern synthesis problem. The proposed method can include the mutual coupling and platform effects by using vectorial active element patterns of an antenna array. A set of synthesis examples with different array geometries and radiation requirements are conducted to validate the effectiveness and advantages of the proposed method.
\end{abstract}

Index Terms-Array pattern synthesis, shaped power pattern, low cross-polarization, semidefinite relaxation(SDR).

\section{INTRODUCTION}

A NTENNA arrays with shaped power patterns have been widely applied in various radar and communication systems. In the past decades, many advanced methods concerning the synthesis of shaped power patterns have been developed, for example, in [1]- [9]. Due to the complexity with high nonlinearity of this problem, many shaped power pattern synthesis methods deal with only the array factors, and they lack of considering the mutual coupling between practical antenna elements. Some techniques including genetic algorithms [10], differential evolution algorithms [11], iterative convex optimization [12]- [13] and semidefinite relaxation [14], [15], can deal with an arbitrary antenna array model with different element patterns, and they would be able to include the mutual coupling effect in the pattern synthesis procedure by using the active element patterns [16], [17]. However, these techniques used to consider only the shape distribution of the total radiation power and part of radiation power actually corresponds to the electric field in undesired polarization. This issue becomes significant in some applications when the antenna array is required to radiate the electric field in a particular polarization and thus the cross-polarization level (XPL) should be reduced as much as possible [18].

Usually, the XPL suppression can be realized by choosing appropriate element structure and array arrangement. Nevertheless, using

Manuscript received June 13, 2017. This work was supported in part by the Shenzhen Science and Technology Innovation Project under Grant YCYJ20170307141315473 and in part by the Science and Technology Research Project of Fujian Province under Grant 201710017.

Y. Liu, J. Bai, K. D. Xu*, Z. Xu and F. Han are with the Department of Electronic Science, Xiamen University, Xiamen 361005, China, and also with the Shenzhen Research Institute of Xiamen University, Shenzhen 518057, China (*corresponding email: kaidaxu@xmu.edu.cn).

Q. H. Liu is with the Department of Electrical and Computer Engineering, Duke University, Durham, NC 27708, USA.

Y. J. Guo are with the Global Big Data Technologies Centre, University of Technology Sydney, Ultimo, NSW 2007, Australia. optimized element excitations can further reduce the XPL. This is very significant when a shaped pattern with low XPL is required for a given antenna array geometry structure. In recent years, some researchers have paid attention to the pattern synthesis with polarization consideration such as jointly synthesizing focused beam patterns with desired polarization properties in [19]- [22] and designing circularly polarized shaped patterns in [23]- [25]. However, when it comes to the problem of synthesizing a linearly polarized shaped power pattern, one difficulty is that the realizable co-polarization direction of a radiated field always changes with the propagation direction and may be much different from the user-defined desired polarization. To the best of our knowledge, how to synthesize a linearly polarized shaped power pattern with low XPL has been never discussed in literature.

In this work, we introduce a definition of the realizable copolarization (COP) direction which is considered as the projection of the user-defined desired polarization direction onto the wavefront plane that is perpendicular to the propagation direction. For a fixed user-defined desired polarization, the realizable COP direction may change with the propagation direction. With this definition, we can easily express the realizable COP and XP components of an antenna array for an arbitrary propagation direction. Then the semi-definite relaxation (SDR) method is applied to deal with the problem of synthesizing a linearly polarized shaped power pattern with control on the COP power shape in the mainlobe region, XPL in any region of interest as well as the total power level in the sidelobe region. The SDR method can relax the non-convex lower bound constraint of the shaped power pattern in such a way to deal with a related convex problem. This technique has been recently effectively used to deal with the problem of phase-only pattern notching in [26], as well as synthesizing shaped power patterns with phase-only optimization [14] or dynamic amplitude ratio control [15]. Despite the success, the mutual coupling and XP effects of practical arrays have been not included in the consideration of [14], [15] and [26]. Now, with the help of the definitions of realizable COP direction and the concept of vectorial active element pattern, the SDR method is extended to synthesize the linearly polarized shaped power patterns with mutual coupling and XPL suppression for practical arrays. Several synthesis examples for linear, sectorial and cylindrical conformal arrays have been provided, and synthesis results have shown the effectiveness and advantages of the proposed method.

\section{Linearly Polarized Shaped Power Pattern Synthesis METHOD}

\section{A. Vectorial far-field pattern expression}

Consider a general antenna array composed of $N$ elements which are placed at locations $\vec{r}_{n}(n=1,2, \cdots, N)$. The far-field radiation pattern can be expressed as

$$
\vec{E}_{t o l}(\theta, \varphi)=\sum_{n=1}^{N} w_{n}\left[\vec{\theta} E_{n \theta}(\theta, \varphi)+\vec{\varphi} E_{n \varphi}(\theta, \varphi)\right] e^{j k \vec{r}_{n} \cdot \vec{a}(\theta, \varphi)}
$$




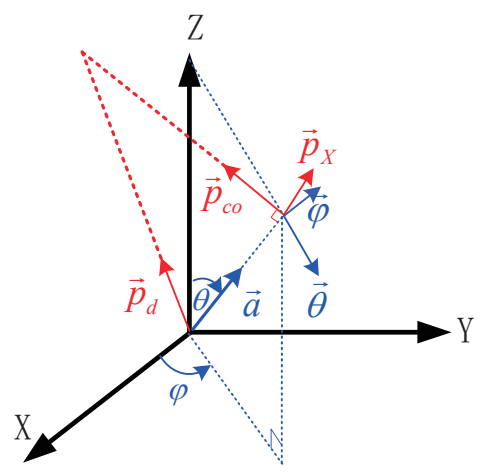

Fig. 1. Illustration of the definitions of realizable COP and XP directions.

where

$$
\begin{gathered}
\vec{\theta}=\cos \theta \cos \varphi \vec{x}+\cos \theta \sin \varphi \vec{y}-\sin \theta \vec{z} \\
\vec{\varphi}=-\sin \varphi \vec{x}+\cos \varphi \vec{y} \\
\vec{a}(\theta, \varphi)=\sin \theta \cos \varphi \vec{x}+\sin \theta \sin \varphi \vec{y}+\cos \theta \vec{z}
\end{gathered}
$$

In the above, $w_{n}$ is the complex excitation of the $n$th element, and $k=2 \pi / \lambda$ where $\lambda$ is the wavelength. $E_{n \theta}(\theta, \varphi)$ and $E_{n \varphi}(\theta, \varphi)$ are the $\vec{\theta}$ and $\vec{\varphi}$-polarization components of the far-field pattern radiated by the $n$th element, respectively. In the array environment, vectorial active element patterns can be chosen to include the mutual coupling and platform effect [17].

Now, by defining the following vectors

$$
\begin{gathered}
\mathbf{s}_{\theta}=\left[E_{1 \theta} e^{j k \vec{r}_{1} \cdot \vec{a}(\theta, \varphi)}, \cdots, E_{N \theta} e^{j k \vec{r}_{N} \cdot \vec{a}(\theta, \varphi)}\right]^{T} \\
\mathbf{s}_{\varphi}=\left[E_{1 \varphi} e^{j k \vec{r}_{1} \cdot \vec{a}(\theta, \varphi)}, \cdots, E_{N \varphi} e^{j k \vec{r}_{N} \cdot \vec{a}(\theta, \varphi)}\right]^{T} \\
\mathbf{w}=\left[w_{1}, w_{2}, \cdots, w_{N}\right]^{T},
\end{gathered}
$$

we can rewrite (1) as

$$
\vec{E}_{t o l}(\theta, \varphi)=\vec{\theta} \mathbf{s}_{\theta}^{H} \mathbf{w}+\vec{\varphi} \mathbf{s}_{\varphi}^{H} \mathbf{w}
$$

where the superscript ' $H$ ' denotes the Hermitian transposition of a matrix.

\section{B. Definitions of the realizable COP and XP directions}

In general, the user-defined desired polarization direction is known a priori and it is determined depending on the practical applications. However, the realizable COP direction radiated by the antenna array is always perpendicular to the propagation direction $\vec{a}(\theta, \varphi)$, and it usually varies with the changing of $\vec{a}(\theta, \varphi)$. Hence, if we look at the realizable COP in wide-angle space, it is in general different from the user-defined desired polarization which is usually given as one fixed direction. Assume that $\vec{p}_{d}$ denotes the user-defined desired polarization direction. For the propagation direction $(\theta, \varphi)$, the projection of $\vec{p}_{d}$ onto the wavefront plane that is perpendicular to the direction $(\theta, \varphi)$ can be considered as the realizable COP direction, as shown in Fig. 1. Mathematically, the normalized realizable COP direction $\vec{p}_{c o}$ can be given as

$$
\vec{p}_{c o}=\frac{\vec{p}_{d}-\left[\vec{p}_{d} \cdot \vec{a}(\theta, \varphi)\right] \vec{a}(\theta, \varphi)}{\left|\vec{p}_{d}-\left[\vec{p}_{d} \cdot \vec{a}(\theta, \varphi)\right] \vec{a}(\theta, \varphi)\right|}
$$

The realizable XP direction $\vec{p}_{X}$ should be perpendicular to both $\vec{p}_{c o}$ and $\vec{a}(\theta, \varphi)$. Thus $\vec{p}_{X}$ is given by

$$
\vec{p}_{X}=\vec{p}_{c o} \times \vec{a}(\theta, \varphi)
$$

Once the realizable COP and XP directions are defined, the COP and XP components of the radiated pattern can be given by

$$
\begin{gathered}
\vec{E}_{c o}(\theta, \varphi)=\left[\vec{E}_{t o l}(\theta, \varphi) \cdot \vec{p}_{c o}\right] \vec{p}_{c o}=\vec{p}_{c o} \mathbf{s}_{c o}^{H} \mathbf{w} \\
\vec{E}_{X}(\theta, \varphi)=\left[\vec{E}_{t o l}(\theta, \varphi) \cdot \vec{p}_{X}\right] \vec{p}_{X}=\vec{p}_{X} \mathbf{s}_{X}^{H} \mathbf{w}
\end{gathered}
$$

respectively, where

$$
\begin{aligned}
& \mathbf{s}_{c o}=\left(\vec{\theta} \cdot \vec{p}_{c o}\right) \mathbf{s}_{\theta}+\left(\vec{\varphi} \cdot \vec{p}_{c o}\right) \mathbf{s}_{\varphi} \\
& \mathbf{s}_{X}=\left(\vec{\theta} \cdot \vec{p}_{X}\right) \mathbf{s}_{\theta}+\left(\vec{\varphi} \cdot \vec{p}_{X}\right) \mathbf{s}_{\varphi} .
\end{aligned}
$$

\section{Shaped pattern synthesis with XPL control}

Our goal is to find appropriate excitation weights so as to produce a linearly polarized shaped power pattern which meets the following constraints: a) the ripple of the COP component is restricted by the upper bound $U_{M L}(\theta, \varphi)$ and the lower bound $L_{M L}(\theta, \varphi)$ over the shaped mainlobe region $\Omega_{M L}$; b) the XPL is required to be less than $\Gamma_{X}$ for the region of interest $\Omega_{X}$; c) the total power pattern level should be less than $\Gamma_{S L}$ in the sidelobe region $\Omega_{S L}$. The constraints can be written:

$$
\begin{cases}L_{M L}(\theta, \varphi) \leq\left|\vec{E}_{c o}(\theta, \varphi)\right|^{2} \leq U_{M L}(\theta, \varphi), & (\theta, \varphi) \in \Omega_{S B} \\ \left|\vec{E}_{X}(\theta, \varphi)\right|^{2} \leq \Gamma_{X}, & (\theta, \varphi) \in \Omega_{X} \\ \left|\vec{E}_{t o l}(\theta, \varphi)\right|^{2} \leq \Gamma_{S L}, & (\theta, \varphi) \in \Omega_{S L}\end{cases}
$$

In the following, the argument $(\theta, \varphi)$ are dropped for notation simplicity. By defining

$$
\begin{gathered}
\mathbf{S}_{c o}=\left[\begin{array}{cc}
\operatorname{Re}\left(\mathbf{s}_{c o}\right) & -\operatorname{Im}\left(\mathbf{s}_{c o}\right) \\
\operatorname{Im}\left(\mathbf{s}_{c o}\right) & \operatorname{Re}\left(\mathbf{s}_{c o}\right)
\end{array}\right] \\
\widetilde{\mathbf{w}}=\left[\begin{array}{c}
\operatorname{Re}(\mathbf{w}) \\
\operatorname{Im}(\mathbf{w})
\end{array}\right]
\end{gathered}
$$

we obtain that

$$
\left|\vec{E}_{c o}\right|^{2}=\widetilde{\mathbf{w}}^{T} \mathbf{Q}_{c o} \widetilde{\mathbf{w}}, \text { with } \mathbf{Q}_{c o}=\mathbf{S}_{c o}^{T} \mathbf{S}_{c o}
$$

Similarly, we have

$$
\begin{aligned}
\left|\vec{E}_{X}\right|^{2} & =\widetilde{\mathbf{w}}^{T} \mathbf{Q}_{X} \widetilde{\mathbf{w}}, \text { with } \mathbf{Q}_{X}=\mathbf{S}_{X}^{T} \mathbf{S}_{X} \\
\left|\vec{E}_{t o l}\right|^{2} & =\widetilde{\mathbf{w}}^{T} \mathbf{Q}_{t o l} \widetilde{\mathbf{w}}, \text { with } \mathbf{Q}_{t o l}=\mathbf{S}_{t o l}^{T} \mathbf{S}_{t o l}
\end{aligned}
$$

where

$$
\begin{gathered}
\mathbf{S}_{X}=\left[\begin{array}{cc}
\operatorname{Re}\left(\mathbf{s}_{X}\right) & -\operatorname{Im}\left(\mathbf{s}_{X}\right) \\
\operatorname{Im}\left(\mathbf{s}_{X}\right) & \operatorname{Re}\left(\mathbf{s}_{X}\right)
\end{array}\right] \\
\mathbf{S}_{t o l}=\left[\begin{array}{cc}
\operatorname{Re}\left(\mathbf{s}_{\theta}\right) & -\operatorname{Im}\left(\mathbf{s}_{\theta}\right) \\
\operatorname{Im}\left(\mathbf{s}_{\theta}\right) & \operatorname{Re}\left(\mathbf{s}_{\theta}\right) \\
\operatorname{Re}\left(\mathbf{s}_{\varphi}\right) & -\operatorname{Im}\left(\mathbf{s}_{\varphi}\right) \\
\operatorname{Im}\left(\mathbf{s}_{\varphi}\right) & \operatorname{Re}\left(\mathbf{s}_{\varphi}\right)
\end{array}\right] .
\end{gathered}
$$

It is well-known that for any real symmetric matrix $\mathbf{Q}$ and any real vector $\widetilde{\mathbf{w}}$, there exists

$$
\widetilde{\mathbf{w}}^{T} \mathbf{Q} \widetilde{\mathbf{w}}=\operatorname{Tr}\left(\widetilde{\mathbf{w}}^{T} \mathbf{Q} \widetilde{\mathbf{w}}\right)=\operatorname{Tr}(\mathbf{Q W})
$$

where $\mathbf{W}=\widetilde{\mathbf{w}} \widetilde{\mathbf{w}}^{T}$ is a rank-one symmetric positive semidefinite matrix. That is, $\mathbf{W} \succeq 0$ and $\operatorname{rank}(\mathbf{W})=1$. Now, the linearly polarized shaped pattern synthesis problem can be reformulated as: find $\mathbf{W}$ such that

$$
\begin{cases}\operatorname{Tr}\left(\mathbf{Q}_{c o} \mathbf{W}\right) \geq L_{M L}(\theta, \varphi), & (\theta, \varphi) \in \Omega_{M L} \\ \operatorname{Tr}\left(\mathbf{Q}_{c o} \mathbf{W}\right) \leq U_{M L}(\theta, \varphi), & (\theta, \varphi) \in \Omega_{M L} \\ \operatorname{Tr}\left(\mathbf{Q}_{X} \mathbf{W}\right) \leq \Gamma_{X}, & (\theta, \varphi) \in \Omega_{X} \\ \operatorname{Tr}\left(\mathbf{Q}_{S L} \mathbf{W}\right) \leq \Gamma_{S L}, & (\theta, \varphi) \in \Omega_{S L} \\ \mathbf{W} \succeq 0, \operatorname{rank}(\mathbf{W})=1 & \end{cases}
$$


The constraint of $\operatorname{rank}(\mathbf{W})=1$ is typically non-convex, and consequently the above problem cannot be solved by using convex optimization algorithms. Hence, we are going to drop this constraint, and choose to iteratively minimize the rank of $\mathbf{W}$ instead. That is,

$$
\begin{aligned}
& \mathbf{W}_{k+1}=\underset{\mathbf{W} \in \mathbb{C}}{\operatorname{argmin}} \operatorname{Tr}\left(\mathbf{W}_{k}+\delta I\right)^{-1} \mathbf{W} \\
& \text { Const. } \begin{cases}\operatorname{Tr}\left(\mathbf{Q}_{c o} \mathbf{W}\right) \geq L_{M L}(\theta, \varphi), & (\theta, \varphi) \in \Omega_{M L} \\
\operatorname{Tr}\left(\mathbf{Q}_{c o} \mathbf{W}\right) \leq U_{M L}(\theta, \varphi), & (\theta, \varphi) \in \Omega_{M L} \\
\operatorname{Tr}\left(\mathbf{Q}_{X} \mathbf{W}\right) \leq \Gamma_{X}, & (\theta, \varphi) \in \Omega_{X} \\
\operatorname{Tr}\left(\mathbf{Q}_{S L} \mathbf{W}\right) \leq \Gamma_{S L}, & (\theta, \varphi) \in \Omega_{S L} \\
\mathbf{W} \succeq 0 & \end{cases}
\end{aligned}
$$

where $\delta>0$ is a small regularization parameter [27]. Such a relaxation is called the semi-definite relaxation (SDR). Now, the problem can be efficiently solved by performing convex optimization. Once the optimal solution of $\mathbf{W}$ is achieved, the $\widetilde{\mathbf{w}}$ vector can be recovered via an eigenvalue decomposition:

$$
\mathbf{W} \approx \sigma_{\max } \mathbf{v} \mathbf{v}^{T}
$$

where $\sigma_{\max }$ is the largest eigenvalue of $\mathbf{W}$ and $\mathbf{v}$ is the corresponding eigenvector. Then, we obtain $\widetilde{\mathbf{w}}=\sqrt{\sigma}_{\max } \mathbf{v}$. Note that since $\operatorname{rank}(\mathbf{W})=1$ cannot be exactly satisfied due to the relaxation operation, the decomposition of (24) may have an approximation error. To improve the accuracy, we can iteratively reduce the rank of $\mathbf{W}$ by following the procedure of (23) until the rank is close enough to 1 or the largest eigenvalue is several order of magnitude larger than the second one. In the iteration, the parameter $\delta$ should be much smaller than $\sigma_{\max }$, and in the examples given below we choose $\delta=\sigma_{\max } / 10$ where $\sigma_{\max }$ is obtained at the previous iteration.

\section{NUMERICAL EXAMPLES}

In this section, four numerical examples for synthesizing linearly polarized shaped patterns with control of both COP and XP components for different antenna array geometries are presented to illustrate the effectiveness and advantages of the proposed method. The concept of active element pattern (AEP) is used to include the mutual coupling as well as the platform effect, and all the AEPs for each tested array are obtained by using High Frequency Structure Simulator (HFSS) software.

\section{A. Cosecant pattern with XPL control for a linear array}

As the first example, we consider to synthesize a linearly polarized cosecant pattern for a 16-element linear array working at $10 \mathrm{GHz}$. As shown in Fig. 2, this array consists of 16 microstrip antennas with a uniform spacing of $15 \mathrm{~mm}$ (i.e., $0.5 \lambda$ at $10 \mathrm{GHz}$ ). Assume that the user-defined desired polarization $\vec{p}_{d}=\vec{y}$. According to (9) and (10), $\vec{p}_{c o}$ coincides with $\vec{\varphi}$ and $\vec{p}_{X}=\vec{\theta}$ on the XOZ plane. For comparison, at first we use the method presented in [1] to synthesize a cosecant-squared array factor without considering mutual coupling. The obtained array factor is satisfactory within the prescribed lower and upper bounds. However, the realized array pattern varing considerably, is beyond the desired bounds, as show in Fig. 3. Especially, for the $-30 \mathrm{~dB}$ upper bound in the null region, the actual SLL is increased to $-27.2 \mathrm{~dB}$ due to practical mutual coupling. The XPL is about $-27 \mathrm{~dB}$ in this situation. Now, we apply the proposed method to this array. We get the $\vec{\theta}$ - and $\vec{\varphi}$-polarization AEPs for each antenna element from the HFSS full-wave simulation. The COP component is restricted by the upper and lower bounds, and the XPL is set to be less than $-30 \mathrm{~dB}$. The synthesized COP and XP patterns are also shown in Fig. 3 for comparison. As can be seen, the synthesized COP pattern completely meets the preset bounds even in the null region, and the XPL also reaches the requirement. Note that the current synthesized results have included the element mutual

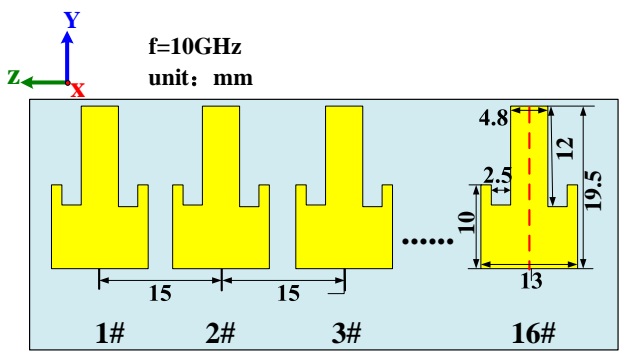

Fig. 2. Geometry of a 16-element microstrip antenna array (a substrate with $\epsilon_{r}=2.2$ and thickness of $1.57 \mathrm{~mm}$ is used).

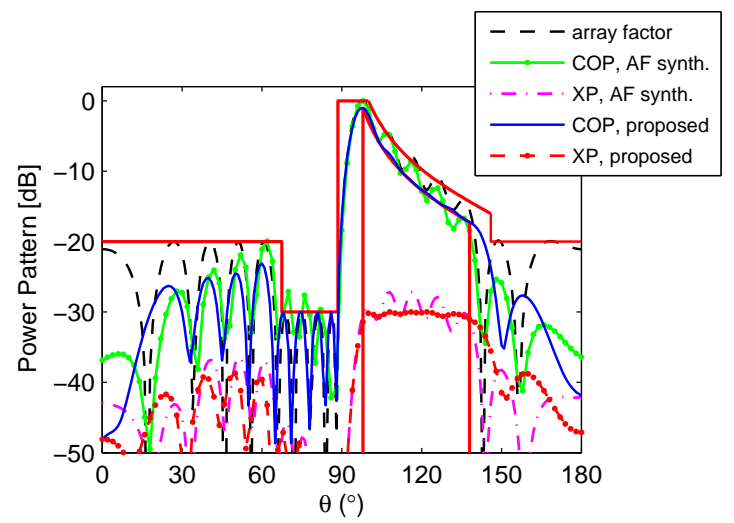

Fig. 3. The synthesized COP and XP patterns for the linear array by the proposed method with accurate vectorial pattern control and the array factor synthesis method in [1].

coupling. Fig. 4 shows the comparison of the excitation distributions obtained by the proposed method and the method in [1].

\section{B. Flap-top pattern with XPL control for a sectorial array}

The second example is to synthesize a linearly polarized flaptop power pattern for a sectorial conformal array with 16 elements which are uniformly distributed on an arc with radius of $242.75 \mathrm{~mm}$. Inspired by the planar E-type microstrip antenna in [28], we design a curved E-type microstrip antenna conforming with the arc surface as the array element. The E-type patch is printed on the top side of a thin dielectric layer with thickness of $0.254 \mathrm{~mm}$ and relative permittivity of 2.2. All the elements are uniformly spaced with the interval of $7.5^{\circ}$, and thus the interelement spacing is $31.78 \mathrm{~mm}$ that is about $0.53 \lambda$ at the working frequency of $5 \mathrm{GHz}$. Fig. 5 shows the antenna array geometry. Here, we consider synthesizing a linearly polarized flap-top pattern in the XOZ plane. Still set the user-defined desired polarization $\vec{p}_{d}=\vec{y}$. Thus, the realizable COP and XP directions on XOZ plane are $\vec{\varphi}$ and $\vec{\theta}$, respectively. Assume that the desired COP pattern has a flat-top shape with a ripple of $\pm 0.5 \mathrm{~dB}$ for the region

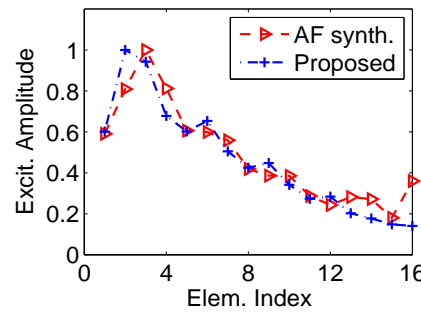

(a)

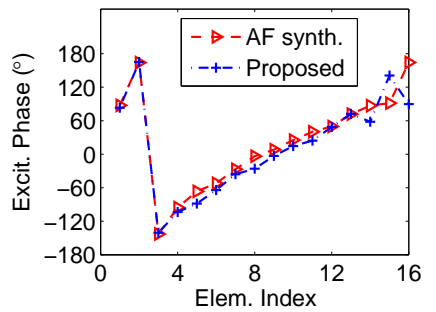

(b)
Fig. 4. The synthesized excitation distributions (corresponding to the patterns in Fig. 3) by the proposed method and the method in [1]. (a) Excitation amplitudes, and (b) excitation phases. 


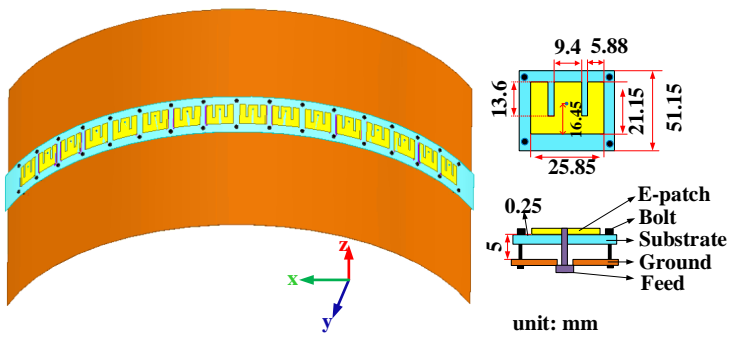

Fig. 5. Geometry of a 16-element sectorial conformal array (with view of the E-type antenna element on planar surface).

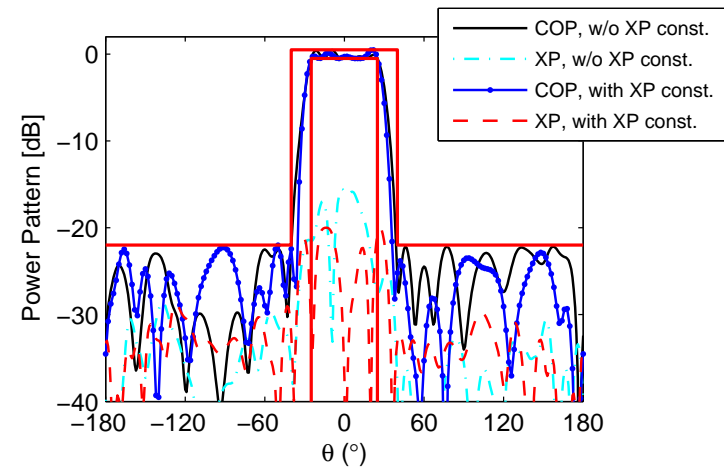

Fig. 6. The obtained COP and XP patterns for the sectorial array by the proposed method with/without a $-20 \mathrm{~dB}$ XPL constraint.

$|\theta| \leq 25^{\circ}$, and the total power pattern level is less than $-22 \mathrm{~dB}$ in the sidelobe region of $|\theta| \geq 40^{\circ}$. For comparison, we at first do not use the XPL constraint, and then obtain the synthesized COP and XP patterns as shown in Fig. 6. As can be seen, the synthesized COP pattern completely meets the prescribed mainlobe bounds and the SLL level also meets the upper bound. Without the XPL constraint, the obtained maximum XPL is $-15.1 \mathrm{~dB}$. Now, we assume an additional constraint that the maximum XPL is no larger than -20 $\mathrm{dB}$ in the whole range of $\theta$. The obtained pattern results are also shown in Fig. 6 for comparison. We can see that the synthesized COP pattern still meets the prescribed bounds, and the maximum XPL is now considerably reduced to $-20 \mathrm{~dB}$. That is, the XPL is reduced by $4.9 \mathrm{~dB}$ by using the additional XPL constraint. In addition, we check what happens if there is one element damaged with no feeding (just connected to matching load). Fig. 7 shows the pattern of the sectorial array if the 9th element is not excited. As can be seen, the performance of the damaged array pattern deteriorates considerably in all of the COP mainlobe, SLL and the XPL. Nevertheless, such a pattern can be corrected by re-optimizing the excitations of the left available elements with the proposed method. The corrected pattern is also shown in Fig. 7, which becomes now satisfying the prescribed bounds again. It should be mentioned that re-optimizing the excitations of the left available elements will certainly improve the performance of a damaged array pattern, but it is in general not guaranteed that the corrected pattern can exactly meet the original prescribed bounds. Fig. 8 shows the comparison of the synthesized excitations by the proposed method with/without XPL constraint as well as the re-optimized excitations for the damaged array.

\section{Circular flap-top pattern with XPL control for a $12 \times 6$-element cylindrical array}

In order to further validate the effectiveness of the proposed method, we construct a $12 \times 6$-element cylindrical conformal array. As shown in Fig. 9, the array elements are planar E-type patch antennas working at $2.4 \mathrm{GHz}$ [28], and they are uniformly distributed with

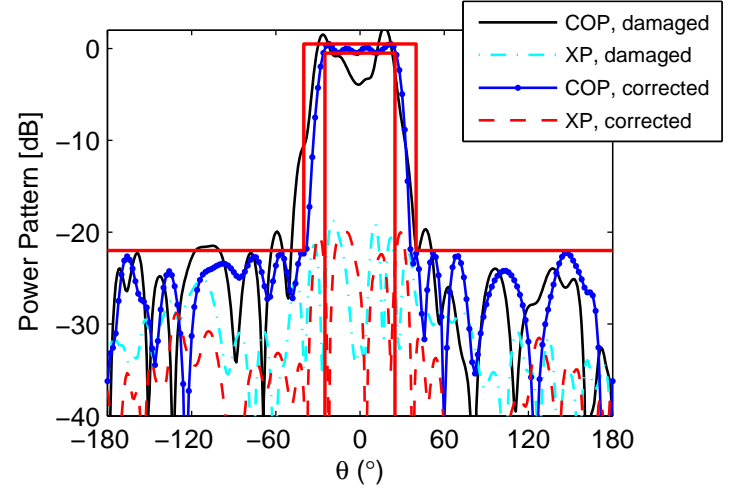

Fig. 7. The obtained COP and XP patterns of the damaged sectorial array (the 9th element is not excited) and the corrected COP and XP patterns by re-optimizing the excitations of the left elements.

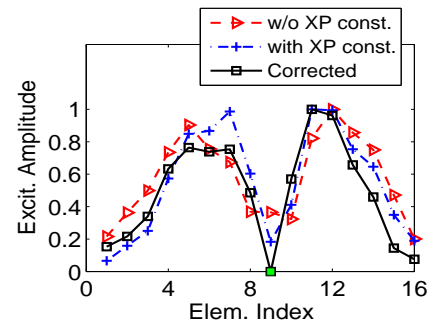

(a)

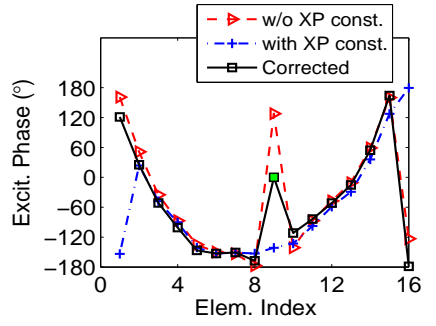

(b)
Fig. 8. The synthesized excitation distributions by the proposed method with/without XPL constraint as well as the corrected excitations for the damaged array (the 9th element is not excited).(a) Excitation amplitudes, and (b) excitation phases.

an interval of $10^{\circ}$ along in circle and a spacing of $62.5 \mathrm{~mm}$ along in cylinder height direction. Assume that the use-defined desired direction is still $\vec{y}$-direction. Note that the realizable COP and XP directions change with the propagation direction $(\theta, \varphi)$. Fig. 10 shows the calculated $\vec{p}_{c o}$ and $\vec{p}_{X}$ according to (9) and (10) for different propagation directions in the semispherical space of $z \geq 0$. Clearly, $\vec{p}_{c o}$ and $\vec{p}_{X}$ are no longer $\vec{\varphi}$ and $\vec{\theta}$ when the propagation direction do not lie on the XOZ plane. The constraints are used as follows: set $-2 \mathrm{~dB} \leq\left|\vec{E}_{c o}(\theta, \varphi)\right|^{2} \leq 0 \mathrm{~dB}$ in the shaped region of $\{|\theta| \leq$ $16^{\circ}$ and $\left.\varphi \in\left(0^{\circ}, 360^{\circ}\right)\right\}$, and then set $\left|\vec{E}_{t o l}(\theta, \varphi)\right|^{2} \leq-20 \mathrm{~dB}$ in the sidelobe region of $\left\{|\theta| \geq 32^{\circ}\right.$ and $\left.\varphi \in\left(0^{\circ}, 360^{\circ}\right)\right\}$. In this situation, the XPL constraint is not used. Fig. 11(a) shows the $\varphi$ cuts (in step of $1^{\circ}$ ) of the synthesized COP pattern together with the prescribed bounds, and Fig. 11(b) shows the $\varphi$-cuts of the XP pattern in the whole space. As can be seen, the achieved maximum XPL is $-16.7 \mathrm{~dB}$ in this situation. Then, we add a XPL constraint of $\left|\vec{E}_{X}(\theta, \varphi)\right|^{2} \leq-20 \mathrm{~dB}$ in the semispherical space, and keep the other constraints unchanged. Fig. 11(c) and (d) show the synthesized $\varphi$-cuts of COP and XP patterns, respectively. As can be seen, the obtained COP pattern is still satisfactory, and the XPL reaches the $-20 \mathrm{~dB}$ requirement. This means that the proposed method can reduce the XPL in wide angle space, and consequently improve the linear polarization purity of the synthesized shaped pattern.

\section{Crescent-shaped pattern with XPL control for a $12 \times 12$-element cylindrical array}

In the last example, we consider synthesizing a more complicated shaped pattern with a $12 \times 12$-element conformal array which is obtained by simply doubling the elements of the array in the third example along the cylinder height direction. Assume that all the configurations are the same as those in the third example except that the desired COP mainlobe is now set as $-2 \mathrm{~dB} \leq\left|\vec{E}_{c o}(\theta, \varphi)\right|^{2} \leq$ 


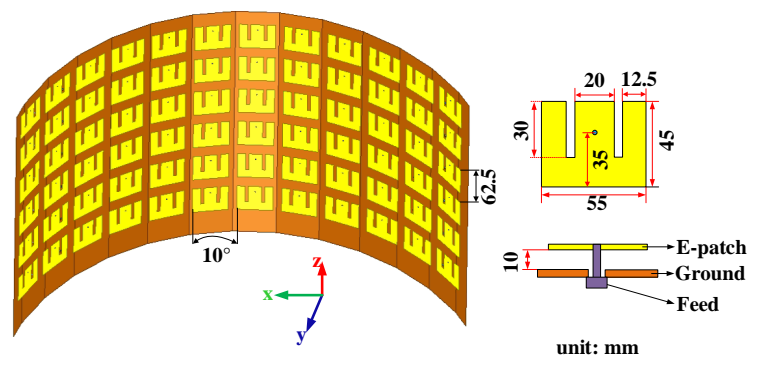

Fig. 9. Geometry of a cylindrical array with $12 \times 6$ E-type patch antennas.

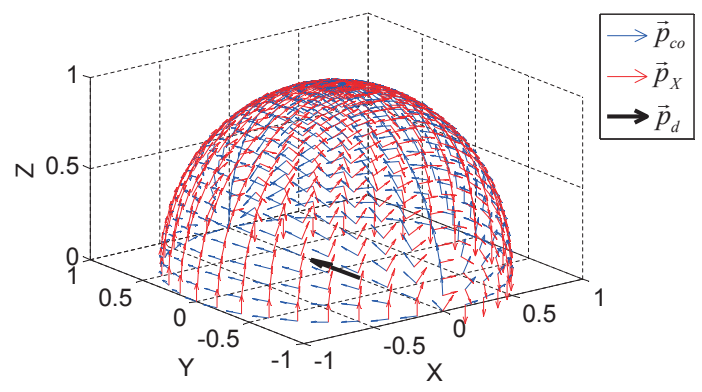

Fig. 10. Illustration of the realizable $\vec{p}_{c o}$ and $\vec{p}_{X}$ when $p_{d}=\vec{y}$.

$0 \mathrm{~dB}$ in the crescent-shaped region of $\left\{(u, v) ; \mid(u+0.35)^{2}+v^{2} \geq\right.$ $\left.0.5^{2} \& u^{2}+v^{2} \leq 0.35^{2}\right\}$ (where $u=\sin \theta \cos \phi$ and $u=\sin \theta \sin \phi$ ), and the SLL is set as $\left|\vec{E}_{t o l}(\theta, \varphi)\right|^{2} \leq-20 \mathrm{~dB}$ in the sidelobe region of $\left\{(u, v) ; \mid(u+0.5)^{2}+v^{2} \leq 0.4^{2}\right.$ or $\left.u^{2}+v^{2} \geq 0.6^{2}\right\}$. Fig. 12 shows top views of the synthesized COP and XP patterns by the proposed method with/without a $-20 \mathrm{~dB}$ XPL constraint. Both of the synthesized COP patterns with/without the XPL constraint do have a crescent-shaped mainlobe and $-20 \mathrm{~dB}$ SLL as expected, while the maximum XPL obtained is $-20 \mathrm{~dB}$ and $-14.98 \mathrm{~dB}$ for the two cases, respectively. This further proves the effectiveness and robustness of the proposed method for different mainlobe shape requirements.

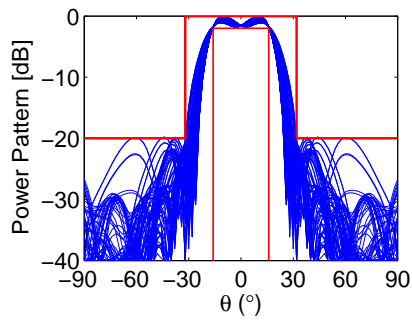

(a)

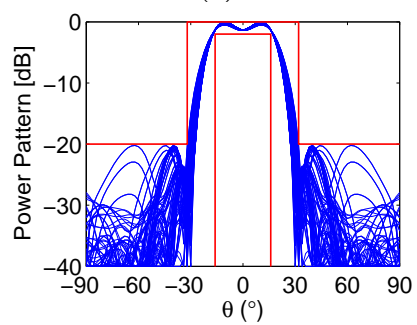

(c)

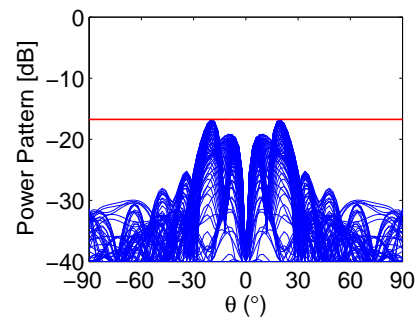

(b)

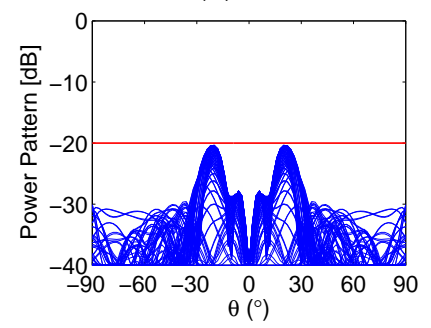

(d)
Fig. 11. The $\varphi$-cuts (in step of $1^{\circ}$ ) of the synthesized circular flat-top patterns for the $12 \times 6$-element cylindrical array by the proposed method. (a) The COP pattern without XPL constraint, (b) the XP pattern without XPL constraint, (c) the COP pattern with a $-20 \mathrm{~dB}$ XPL constraint, and (d) the XP pattern with a $-20 \mathrm{~dB}$ XPL constraint.

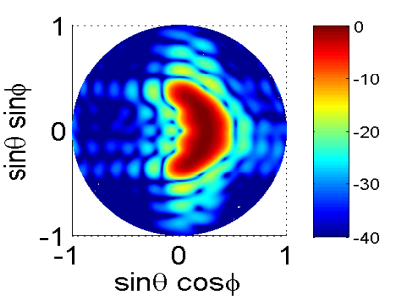

(a)

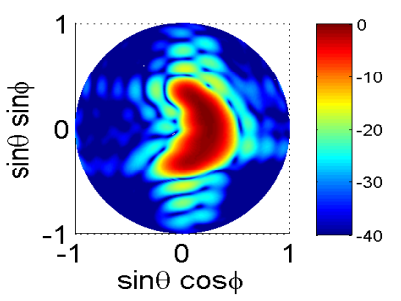

(c)

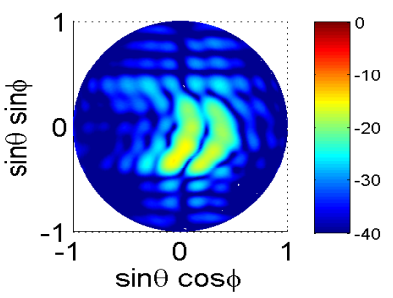

(b)

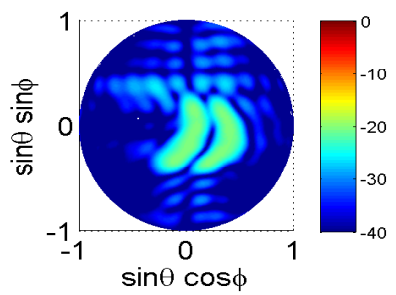

(d)
Fig. 12. Top views of the synthesized crescent-shaped patterns for the $12 \times$ 12 -element cylindrical array by the proposed method. (a) The COP pattern without XPL constraint, (b) The XP pattern without XPL constraint, (c) the COP pattern with a $-20 \mathrm{~dB}$ XPL constraint, and (d) the XP pattern with a $-20 \mathrm{~dB}$ XPL constraint.

\section{Measurement Results}

A prototype of the 16-element sectorial conformal array used in the second example is fabricated, as shown in Fig. 13. A feeding network based on microstrip line is designed to provide the required amplitude and phase distributions given in Fig. 8 for the flat-top pattern with $-22 \mathrm{~dB}$ SLL and $-20 \mathrm{~dB}$ XPL constraint. The $16 \mathrm{E}-$ type patches are printed on a Taconic dielectric with thickness of $0.254 \mathrm{~mm}$ and relative permittivity of 2.2. This dielectric is thin and is easily mounted on the outer side of the cylindrical metal ground with a spacing of $5 \mathrm{~mm}$ by using a number of dielectric screws. The feeding network is curved and clung to the reverse side of the cylindrical ground, and a feeding probe is used which goes through a hole of the ground to connect the microstrip line and the radiating patch of each element. The whole antenna structure is mounted on a dielectric cylinder with the same size as used in simulation. Fig. 14 shows the comparison of the measured COP and XP patterns as well as the synthesized results at $5 \mathrm{GHz}$. As can be seen, the measured COP pattern has almost the same flat-top shape in the mainlobe with a slightly larger ripple though, and the SLL is $-18.8 \mathrm{~dB}$ which is $3.2 \mathrm{~dB}$ higher than the synthesized one. The measured XPL is about $-18.3 \mathrm{~dB}$ that is $1.7 \mathrm{~dB}$ higher than the synthesized one. Despite the performance degradation probably due to fabrication errors and nonideal measurement environment, the measured results in total agree well with the synthesized ones.

\section{CONCLUSION}

In this paper, a novel linearly polarized shaped pattern synthesis method with both cross-polarization and sidelobe level control have been presented. According to the user-defined desired polarization, we introduce the definitions of realizable co- and cross-polarization directions. With the help of such definitions, the linearly polarized shaped pattern synthesis problem is formulated and solved via the semi-definite relaxation scheme. The pattern synthesis experiments have been conducted for different antenna arrays including linear, sectorial, and cylindrical conformal arrays. The synthesis results show that by finding appropriate excitations, the proposed method can obtain satisfactory linearly polarized shaped patterns meeting 


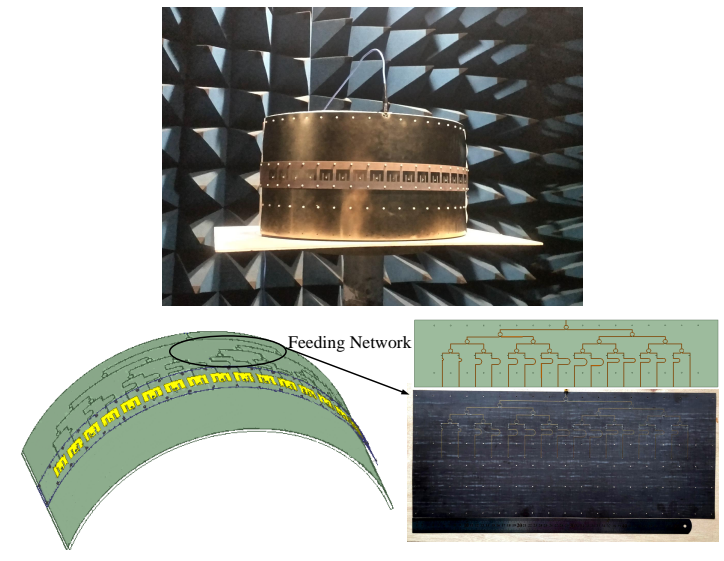

Fig. 13. The fabricated 16-element sectorial conformal array with the feeding network on the reverse side of the ground.

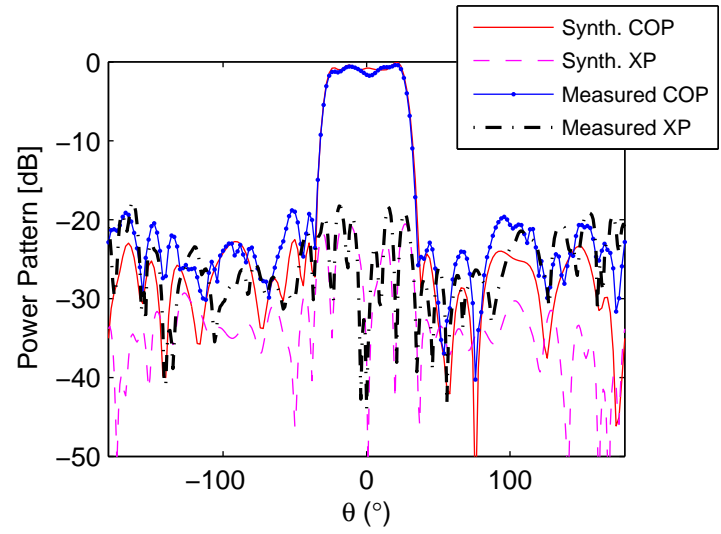

Fig. 14. Comparison of the measured and synthesized patterns of the 16element sectorial conformal array.

the prescribed bounds while reducing the cross-polarization and sidelobe levels considerably. A prototype of sectorial conformal array is fabricated, and the measured co- and cross-polarization patterns are in good agreement with the synthesized results.

Finally, it should be noted that the proposed method is an extension of the semi-definite relaxation (SDR) method which do not directly deal with the original non-convex shaped pattern problem with respect to element excitations, but instead, solves an convex optimization problem in terms of auxiliary variables given by the product amongst the different excitations. Despite the effectiveness, such processing like other SDR methods increases the number of unknowns to be the square of the element count, and consequently it is hard to deal with large-scale array synthesis problems unless highperformance computer source is used. Besides, the proposed method find one single excitation distribution generating a desired linearly polarized shaped power pattern. It should be mentioned that for the same shaped power pattern, multiple different excitation distributions probably exist which provides more degrees of freedom to a designer. The further research in this area would be very interesting.

\section{REFERENCES}

[1] R. S. Elliott and G. J. Stern, "A new technique for shaped beam synthesis of equispaced arrays," IEEE Trans. Antennas Propag., vol. 32, no. 10, pp. 1129-1133, 1984

[2] T. Isernia, O. M. Bucci, and N. Fiorentino, "Shaped beam antenna synthesis problems: feasibility criteria and new strategies," J. Electromagn. Waves and Applicat., vol. 12, no. 1, pp. 103-138, 1998.

[3] F. Wang, V. Balakrishnan, P. Y. Zhou, J. J. Chen, R. Yang, and C. Frank, "Optimal array pattern synthesis using semidefinite programming,"IEEE Trans. Signal Process., vol. 51, no. 5, pp. 1172-1183, 2003.
[4] Y. Liu, Z. Nie, and Q. H. Liu, "A new method for the synthesis of nonuniform linear arrays with shaped power patterns (invited paper)," Prog. Electromagn. Res., vol. 107, pp. 349-363, 2010.

[5] G. Oliveri, M. Carlin, and A. Massa, "Complex-weight sparse linear array synthesis by Bayesian compressive sampling," IEEE Trans. Antennas Propag., vol. 60, no. 5, pp. 2309-2326, 2012.

[6] Y. Liu, Q. H. Liu, and Z. Nie, "Reducing the number of elements in multiple-pattern linear arrays by the extended matrix pencil methods," IEEE Trans. Antennas Propag., vol. 62, no. 2, pp. 652-660, Feb. 2014.

[7] O. M. Bucci, T. Isernia, and A. F. Morabito, "Optimal synthesis of circularly symmetric shaped beams," IEEE Trans. Antennas Propag., vol. 62, no. 4, pp. 1954-1964, Apr. 2014.

[8] A. F. Morabito, A. R. Lagan, G. Sorbello, and T. Isernia, "Maskconstrained power synthesis of maximally sparse linear arrays through a compressive-sensing-driven strategy," J. Electromagn. Waves and Applicat., vol. 29, no. 10, pp. 1384-1396, 2015.

[9] B. Fuchs and S. Rondineau, "Array pattern synthesis with excitation control via norm minimization," IEEE Trans. Antennas Propag., vol. 64, no. 10, pp. 4228-4234, 2016

[10] D. Marcano and F. Duran, "Synthesis of antenna arrays using genetic algorithms," IEEE Antennas Propag. Mag., vol. 42, no. 3, pp. 12-22, 2000.

[11] S. Yang, Y. B. Gan, and P. K. Tan, "A new technique for power-pattern synthesis in time-modulated linear arrays," IEEE Antennas Wireless Propag. Lett., vol. 2, pp. 285-287, 2003.

[12] B. Fuchs. A. Skrivervik, and J. R. Mosig, "Shaped beam synthesis of arrays via sequential convex optimizations," IEEE Antennas Wireless Propag. Lett., vol. 12, pp. 1049-1052, 2013.

[13] K. M. Tsui and S. C. Chan, "Pattern synthesis of narrowband conformal arrays using iterative second-order cone programming," IEEE Trans. Antennas Propag., vol. 58, no. 6, pp. 1959-1970, 2010.

[14] B. Fuchs, "Application of convex relaxation to array synthesis problems," IEEE Trans. Antennas Propag., vol. 62, no. 2, pp. 634-640, 2014.

[15] J. Bai, Y. Liu, J. Cheng, P. You, and Q. H. Liu, "Shaped power pattern antenna array synthesis with reduction of dynamic range ratio," presented at Progress in Electromagnetic Research Symposium (PIERS), Shanghai, China, Aug. 2016.

[16] D. F. Kelly and W. L. Stutzman, "Array antenna pattern modeling methods that include mutual coupling effects," IEEE Trans. Antennas Propag., vol. 41, no. 12, pp. 1625-1632, Dec. 1993.

[17] L. Caccavale, T. Isernia, and F. Soldovieri, "Methods for optimal focusing of microstrip array antennas including mutual coupling," IEE Proc. Microw. Antennas Propag., vol. 147, no. 3, pp. 199-202, 2000.

[18] D. Giuli, "Polarization diversity in radars," Proceedings of the IEEE, vol. 74, no. 2, pp. 245-269, 1986.

[19] B.-H. Wang, Y. Guo, Y. L. Wang, and Y. Z. Lin, "Frequencyinvariant pattern synthesis of conformal array antenna with low crosspolarisation," IET Microw. Antennas Propag., vol. 2, no. 5, pp.442-450, 2008.

[20] J. J. Xiao and A. Nehorai, "Optimal polarized beampattern synthesis using a vector antenna array," IEEE Trans. Signal Process., vol. 57, no. 2 , pp. 576-587, 2009.

[21] B. Fuchs and J. J. Fuchs, "Optimal polarization synthesis of arbitrary arrays with focused power pattern," IEEE Trans. Antennas Propag., vol. 59, no. 12, pp. 4512-4519, 2011.

[22] M. Li, Y. Chang, Y. Li, J. Dong, and X. Wang, "Optimal polarised pattern synthesis of wideband arrays via convex optimisation," IET Microw. Antennas Propag., vol. 7, no. 15, pp. 1228-1237, 2013.

[23] B. Fuchs, "Polarization synthesis of arbitrary arrays with shaped beam pattern," presented at IEEE International Symposium on Phased Array Systems and Technology, Waltham, MA, USA, Oct. 2013, pp. 689-692.

[24] L. I. Vaskelainen, "Iterative least-squares synthesis methods for conformal array antennas with optimized polarization and frequency properties," IEEE Trans. Antennas Propag., vol. 45, no. 7, pp. 1179-1185, 1997.

[25] M. Li, X. Wang, Y. Li, and H. Dai, "Polarization beam pattern synthesis based on particle swarm optimization," IEICE Electronics Express., vol. 9, no. 21, pp. 1648-1653, 2012.

[26] P. J. Kajenski, "Phase only antenna pattern notching via a semidefinite programming relaxation," IEEE Trans. Antennas Propag., vol. 60, no. 5, pp. 2562-2565, 2012.

[27] M. Fazel, H. Hindi, and S. Boyd, "Rank minimization and applications in system theory," presented at the Amer. Contr. Conf. Boston, MA, USA, Jun. 2004.

[28] F. Yang, X. X. Zhang, X. Ye, and Y. Rahmat Samii, "Wideband E-shaped patch antennas for wireless communications," IEEE Trans. Antennas Propag., vol. 49, no. 7, pp. 1094-1100, 2001. 